\title{
Vacunación sublingual con bacterias inactivadas en pacientes nefrológicos con infección urinaria recurrente: experiencia en un centro de España
} Sublingual vaccination with inactivated bacteria in recurrent urinary tract infection of nephrologic patients: experience in a center in Spain

\author{
${ }^{\oplus}$ Rebeca García Agudo ${ }^{1}{ }^{\oplus}$ Beatriz Proy Vega ${ }^{2},{ }^{\odot}$ Ángel Arias Arias ${ }^{3},{ }^{\oplus}$ Nayara Panizo González ${ }^{1}$, \\ ${ }^{\oplus}$ Fátima Cazalla Cadenas ${ }^{1}$, $\odot$ Elisa Berta Pereira Pérez ${ }^{1},{ }^{\oplus}$ Olga Redondo González . \\ ${ }^{1}$ Servicio de Nefrología, Hospital La Mancha-Centro, Alcázar de San Juan, Ciudad Real, España. \\ ${ }^{2}$ Servicio de Farmacia Hospitalaria, Hospital La Mancha-Centro, Alcázar de San Juan, Ciudad Real, España. \\ ${ }^{3}$ Unidad de Apoyo a la Investigación, Hospital La Mancha-Centro, Alcázar de San Juan, Ciudad Real, España. \\ ${ }^{4}$ Servicio de Medicina Preventiva, Hospital Universitario de Guadalajara, Guadalajara, España.
}

\begin{abstract}
Resumen
Introducción: las infecciones del tracto urinario (ITU) son frecuentes en pacientes con enfermedad renal crónica (ERC). Una opción de tratamiento cuando estas infecciones son recurrentes es la vacunación bacteriana sublingual.

Objetivo: determinar la respuesta a la vacunación bacteriana sublingual en pacientes nefrológicos con ITU recurrente.

Materiales y métodos: estudio cuasi experimental en el que se evaluó la evolución en 15 meses de los pacientes con ITU recurrente que asistieron a consulta externa de nefrología. Tras recibir tratamiento antibiótico según antibiograma para cada ITU, los participantes tomaron un ciclo de la vacuna sublingual bacteriana Uromune ${ }^{\circledR}$ durante tres meses. Se recogieron datos sociodemográficos y sobre factores de riesgo asociados, análisis de sangre y orina, episodios de ITU en los seis meses previos y posteriores, microorganismos causantes, tratamiento antibiótico concomitante, respuesta al tratamiento y resolución de la ITU.

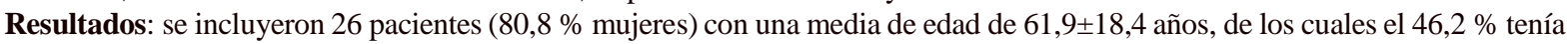

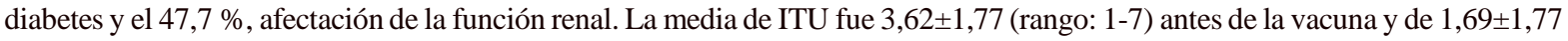
(rango: 0-5) después. Se recogieron 184 urocultivos: 74,9 \% positivos, 16,9\% negativos y 8,2 \% contaminados. Las bacterias más frecuentes fueron Escherichia coli (55,4 \%), Enterococcus faecalis (6 \%) y Enterobacter cloacae (2,7 \%). El $50 \%$ de los participantes presentó síndrome miccional, que se asoció inversamente con la edad ( $\mathrm{p}<0,05)$. El 26,9 \% no volvió a tener ITU y el 73,1 \% tuvo menos episodios. Los pacientes con ERC avanzada (estadios IV-V) respondieron peor a la vacuna $(92,9 \%$ vs $50 \%, \mathrm{p}=0,025)$. Conclusiones: la vacunación bacteriana sublingual es una buena opción de tratamiento para la ITU recurrente de pacientes con ERC, siendo más eficaz en los que presentan mejor función renal.
\end{abstract}

Palabras clave: Insuficiencia renal crónica, infecciones urinarias, vacunas bacterianas (DeCS).

doi:http://dx.doi.org/10.22265/acnef.7.2.387

\begin{abstract}
Introduction: Urinary tract infections (UTIs) are common in patients with chronic kidney disease. A treatment option in recurrent UTI is sublingual bacterial vaccination. The objective of this study was to determine the response to vaccination in nephrologic patients with recurrent UTI.

Method: Quasi-experimental study before-after (15 months) in patients with recurrent UTI from the outpatient nephrology consultation. After receiving antibiotic treatment for each UTI, patients took one cycle of the sublingual bacterial vaccine Uromune? for three months. Sociodemographic data, associated risk factors, analysis, UTI in the previous and subsequent six months, microorganisms, concomitant antibiotic treatment, response to treatment and resolution of UTI were collected.

Results: Twenty-six patients ( $80.8 \%$ female) of $61.9 \pm 18.4$ years, $46.2 \%$ with diabetes and $47.7 \%$ with impaired renal function were included. The episodes of UTI were $3.62 \pm 1.77$ (1-7) before and $1.69 \pm 1.77$ (0-5) after vaccination. In total, 184 urine cultures were collected: $74.9 \%$ positive, $16.9 \%$ negative and $8.2 \%$ contaminated. The most frequent bacteria were Escherichia coli (55.4\%), Enterococcus faecalis (6\%) and Enterobacter cloacae (2.7\%). Fifty percent had voiding syndrome, which was inversely associated with age ( $<0.05) ; 26.9 \%$ did not have a UTI again and $73.1 \%$ had fewer episodes. Patients with advanced chronic disease (stages $4-5)$ reponded worse to the vaccine $(92.9 \%$ vs $50 \%, \mathrm{p}=0.025)$.

Conclusions: Sublingual bacterial vaccination is a good treatment option in recurrent UTI of nephrologic patients, being more effective in those with better renal function.
\end{abstract}

Keywords: urinary tract infections, chronic kidney disease, bacterial vaccines (MeSH).

doi:http://dx.doi.org/10.22265/acnef.7.2.387

Citación: García Agudo R, Proy Vega B, Arias Arias A, Panizo González N, Cazalla Cadenas F, Pereira Pérez EB, Redondo González O. Vacunación sublingual con bacterias inactivadas en pacientes nefrológicos con infección urinaria recurrente: experiencia en un centro de España. Rev. Colomb. Nefrol. 2020;7(1):78-84. https:// doi.org/10.22265/acnef.7.2.387

Recibido: 20.02.19, Aceptado: 18.09.19, Publicado en línea: 8.02.19

Correspondencia: Rebeca García Agudo,rgagudo@sescam.jccm.es

78 Vacunación sublingual con bacterias inactivadas en pacientes nefrológicos con infección urinaria recurrente: experiencia en un centro de España 


\section{Introducción}

$\mathrm{L}$ as infecciones del tracto urinario (ITU) son frecuentes en la población general, pero su incidencia ha cambiado en los últimos años registrándose un aumento en la prevalencia de la ITU de origen comunitario y una disminución en la de la ITU nosocomial debido a la menor utilización de sondas urinarias y a la sustitución de circuitos abiertos por cerrados ${ }^{1}$. En los pacientes con enfermedad renal crónica (ERC), la ITU constituye el segundo proceso infeccioso más habitual, solo precedido por la infección respiratoria de vías bajas ${ }^{2}$. Aunque las complicaciones asociadas a cualquier infección suponen una fuente importante de morbimortalidad en los pacientes renales, la aparición de este tipo de infecciones en tratamiento renal sustitutivo es tres veces mayor ${ }^{3-7}$.

La aparición de un evento infeccioso se asocia de forma independiente con la progresión hacia la ERC terminal, la isquemia cardiovascular, la insuficiencia cardíaca congestiva y la mortalidad ${ }^{8}$, lo que obliga a llevar a la práctica, en la medida de lo posible, su prevención, diagnóstico precoz y tratamiento eficaz.

Los pacientes con ERC presentan con mayor frecuencia factores de riesgo para la adquisición de diversas infecciones: por un lado, la uremia origina alteraciones de la respuesta humoral, la función linfocitaria, los macrófagos y los polimorfonucleares, y, por el otro, la etiología de la ERC condiciona la normal evacuación de la orina y la integridad de la vía urinaria o implica su manipulación como en el reflujo vesicoureteral, la vejiga neurógena, las válvulas uretrales, el prostatismo, el sondaje vesical, la cateterización renal, la litiasis complicada y la poliquistosis renal.

En algunos casos la diabetes es la causa subyacente tanto de la ERC como de la mayor susceptibilidad de la aparición de ITU y su peor evolución, en especial en pacientes de sexo femenino y de edad avanzada En todo caso, la incidencia de ITU puede aumentar conforme la ERC progresa y se deterioran los mecanismos defensivos frente a la infección. A su vez, en los pacientes renales se dan condiciones especiales, como el riesgo de nefrotoxicidad por antibioterapia, la necesidad de ajuste de fármacos al filtrado glomerular o al tratamiento con diálisis, el pH bajo del medio urinario y las alteraciones del urotelio, que tienden a disminuir la eficacia de los tratamientos contra las ITU y complican su implementación.

Pese al incremento de casos de pacientes con ERC en los últimos años, existen escasas publicaciones sobre la ITU en esta población y sobre las indicaciones y opciones de tratamiento. Frente a la profilaxis antibiótica nocturna en pacientes con ITU recurrente, el uso de la vacunación se postula como una alternativa para reducir las resistencias a los antibióticos y la polimedicación mediante su efecto inmunomodulador y así estimular tanto el sistema inmunológico innato como el adquirido. Desde 2010 se encuentra disponible en España una vacuna bacteriana individualizada (MV 140Uromune ${ }$, Inmunotek, Q-Pharma) que se presenta en dos viales que contienen una suspensión de 10 cuerpos enteros de bacterias inactivadas de los principales organismos causantes de ITU ${ }^{9}$, entre otros: Escherichia coli, Proteus vulgaris, Klebsiella pneumoniae, Enterococcus faecalis, Staphylococcus saprophyticus y Proteus mirabilis. Su administración se realiza en ayunas durante un mínimo de tres meses y consiste en dos pulverizaciones por vía sublingual que se deben mantener durante dos minutos, siendo el sobrante tragado. El contenido de esta vacuna se endosa a las células dendríticas humanas con la capacidad de generar células $\mathrm{T}$ productoras de Th1/Th7 e IL-10 por mecanismos que dependen de las vías mediadas por el gen 88 (MyD88) que está implicado en la diferenciación mieloide y la biosíntesis tirosina quinasa $(\text { Syk })^{10}$.

Dado el panorama, el objetivo del presente estudio fue determinar la respuesta a la vacunación bacteriana sublingual individualizada de pacientes nefrológicos con ITU recurrente, con y sin deterioro de función renal, atendidos en consulta externa por el servicio de nefrología.

\section{Materiales y métodos}

Se realizó un estudio cuasiexperimental antes-después en los pacientes mayores de 18 años diagnosticados de ITU recurrente que asistieron a consulta externa al servicio de nefrología del Hospital La Mancha-Cen- 
tro, Alcázar de San Juan, Ciudad Real, España. Los pacientes fueron incluidos mediante reclutamiento consecutivo.

La ITU recurrente se definió como la presencia de dos episodios de ITU en seis meses o tres episodios en un año. El período de seguimiento fue de 15 meses: seis meses antes de la vacunación, tres meses de vacunación y seis meses después de la vacunación. Se realizó un urocultivo al inicio, cada tres meses y siempre que el paciente alertara de la posible existencia de ITU por síndrome miccional (polaquiuria, disuria, urgencia miccional) o sospecha de ITU (coluria, orina maloliente, dolor suprapúbico, etc.). Ante una ITU en cualquier momento del seguimiento, a los pacientes se les prescribió antibioterapia según antibiograma y se comprobó su resolución con un nuevo urocultivo una semana después de finalizado el tratamiento. Se excluyeron aquellos pacientes con colonización bacteriana.

Los participantes realizaron un ciclo de vacunación con bacterianas inactivadas (Uromune ${ }^{\circledR}$ ) durante tres meses, en ayunas y por vía sublingual, según la ficha del producto. Se les entregó una hoja de instrucciones para asegurar la correcta administración y cumplimiento, y se comprobó la adherencia terapéutica en cada revisión.

Se recogieron los datos sociodemográficos y la información sobre factores de riesgo asociados a la ITU, parámetros analíticos, número de episodios de ITU en los seis meses previos y posteriores a la administración de la vacuna, microorganismos causantes, presencia de tratamiento o profilaxis antibiótica concomitante, toma de concentrado de arándanos rojos, respuesta al tratamiento y resolución de la ITU.

\section{Resultados}

\section{Datos descriptivos de la muestra}

Se incluyeron 26 pacientes (80,8\% mujeres) con una edad media de $61,9 \pm 18,4$ años. El 46,2 \% de los participantes tenía diabetes; el 11,5\%, ERC en estadios I-II; el 19,2 \%, ERC en estadio III, y el 26,9\%, ERC en estadios IV-V. El 42,3\% de los pacientes no tenía datos de ERC (Tabla 1).

\section{Características de la ITU}

Durante los 15 meses de estudio se recogieron datos de 184 urocultivos: $74,9 \%$ positivos, $16,9 \%$ negativos y $8,2 \%$ contaminados. La bacteria más frecuente fue $E$. coli $(55,4 \%)$, seguida de E faecalis $(6 \%)$ y Enterobacter cloacae $(2,7 \%)$. El $50 \%$ de los pacientes refirió síndrome miccional, el cual se relacionó de manera inversa con la edad ( $\mathrm{p}=0,043)$.

\section{Respuesta a la vacunación}

El número de episodios de ITU fue de 3,62 $\pm 1,77$ (rango: 1-7) en los seis meses anteriores a la adminis-

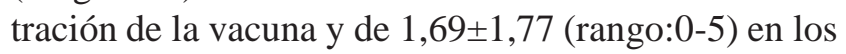
seis meses posteriores $(\mathrm{p}<0,05)$ (Figura 1). El $73 \%$ $(n=26)$ de los pacientes respondió a la vacuna, y de estos el 26,9\% (n=7) no volvió a tener una ITU, el $46,1 \%(\mathrm{n}=12)$ tuvo un menor número de episodios y el $26,9 \%(\mathrm{n}=7)$ no mejoró.

Los pacientes diabéticos respondieron peor a la vacuna en comparación con los que no padecían esta enfermedad (66,7 \% vs. 78,6\%), aunque la diferencia no fue estadísticamente significativa. Los pacientes sin datos de ERC o con estadios I-II de la

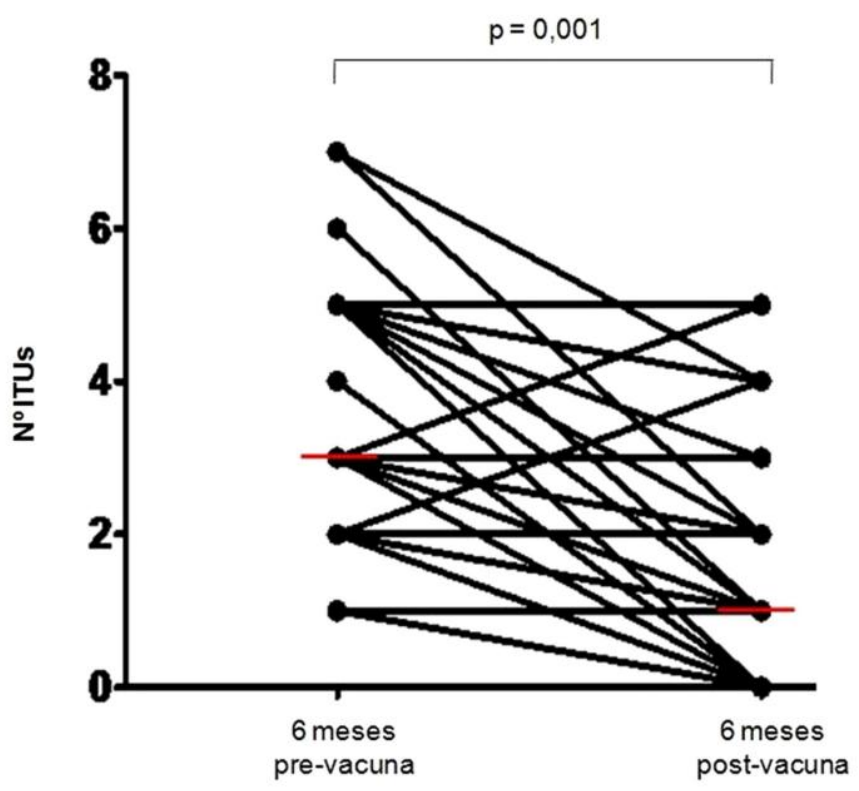

Figura 1. Episodios de infecciones del tracto urinario antes y después de la vacunación.

Fuente: elaboración propia. 
Tabla 1. Características basales de los pacientes.

\begin{tabular}{|c|c|c|}
\hline \multirow{2}{*}{ Sexo } & Hombres & $5(19,2 \%)$ \\
\hline & Mujeres & $21(80,8 \%)$ \\
\hline \multicolumn{2}{|l|}{ Edad (años) } & $61,9 \pm 18,4$ \\
\hline \multirow{5}{*}{ Estadio de enfermedad renal crónica } & Sin datos & $11(42,3 \%)$ \\
\hline & 1 & $1(3,8 \%)$ \\
\hline & 2 & $2(7,7 \%)$ \\
\hline & 3 & $5(19,2 \%)$ \\
\hline & 4 & $7(26,9 \%)$ \\
\hline \multirow{2}{*}{ Antecedentes personales } & Diabetes mellitus & $12(46,2 \%)$ \\
\hline & Inmunosupresión & $5(19,2 \%)$ \\
\hline \multirow{4}{*}{ Urológicos } & Obstrucción urinaria & $4(15,4 \%)$ \\
\hline & Intervencionismo urológico & $4(15,4 \%)$ \\
\hline & Litiasis & $6(23,1 \%)$ \\
\hline & Hipertrofia prostática benigna & $4(80 \%)$ \\
\hline \multirow{6}{*}{ Parámetros analíticos basales } & Hemoglobina (g/dl) & $12,8 \pm 1,9$ \\
\hline & Creatinina (mg/dl) & $1,5 \pm 0,9$ \\
\hline & Urea (mg/dl) & $77,2 \pm 54,3$ \\
\hline & $\begin{array}{l}\text { Filtrado glomerular estimado por MDRD-6 } \\
(\mathrm{ml} / \mathrm{min})\end{array}$ & $56,9 \pm 44,8$ \\
\hline & Albúmina (g/dl) & $4,2 \pm 0,3$ \\
\hline & Proteína C reactiva (mg/dl) & $0,5 \pm 0,5$ \\
\hline
\end{tabular}

MDRD-6: Modification of Diet in Renal Disease, fórmula de 6 parámetros.

Fuente: Elaboración propia.

enfermedad respondieron a la vacunación en un $92,0 \%$ de los casos, mientras que solo el $50 \%$ de los pacientes con ERC en estadios III-IV lo hicieron $(\mathrm{p}=0,026)$. No se encontró correlación entre la respuesta a la vacuna y la edad, el sexo, la etiología de la ERC, los antecedentes urológicos o los parámetros analíticos reseñados.

La aceptación de la vacunación entre los pacientes fue del $100 \%$ y no se observó ninguna intolerancia o evento adverso asociado a la vacuna.

\section{Discusión}

Aunque la ITU recurrente es una patología frecuente, especialmente en mujeres jóvenes, constituye un tema de particular interés en el paciente renal por su comorbilidad y complejidad y porque se relaciona con el riesgo de sepsis, medicación concomitante y alta tasa de hospitalización.

La piedra angular de la ITU recurrente ha sido hasta hoy la profilaxis antibiótica nocturna. Sin embargo, 
debido al aumento de las resistencias bacterianas y el riesgo de interacciones medicamentosas y eventos adversos, existe un interés creciente en la búsqueda de alternativas terapéuticas.

No existen estudios publicados sobre el uso de la vacuna bacteriana en población con ITU y ERC, aunque la administración de preparaciones bacterianas por vía mucosa ha sido previamente utilizada para prevenir la repetición de ITU. En 2002, Bauer et al. ${ }^{11}$ publicaron un metaanálisis de estudios sobre un lisado bacteriano de 18 cepas uropatogénicas de E. coli administrado por vía oral, comparado con placebo en pacientes con ITU (601 mujeres), y demostraron la superioridad de este tratamiento con un intervalo de confianza de 0,64-0,72. Más adelante, en 2005, Bauer et al. ${ }^{12}$ compararon el lisado bacteriano frente a placebo en 454 mujeres, para lo cual suministraron una cápsula (activa o placebo) diaria durante 90 días seguido de tres meses sin tratamiento y un nuevo tratamiento los 10 primeros días de los meses 7, 8 y 9, y demostraron una disminución en el número de episodios de ITU en el $34 \%$ de las pacientes tratadas con el lisado bacteriano.

Por otra parte, Naber et al. ${ }^{13}$ realizaron un metaanálisis de la literatura médica referente a dos vacunas no comercializadas en España (SolcoUrovac®, vaginal, y UroVaxom®, oral) que demostraron ser superiores al placebo: ausencia de ITU recurrente en el $50 \%$ y $14 \%$ de los pacientes, respectivamente.

Con la vacuna MV 140-Uromune®, empleada en el presente trabajo, Lorenzo-Gómez et al. ${ }^{14}$ realizaron un estudio observacional multicéntrico en 319 mujeres con ITU recurrente en el cual 159 participantes recibieron la vacuna durante tres meses y 160 tomaron una profilaxis antibiótica $(40 \mathrm{mg} / 200 \mathrm{mg}$ al día de trimetoprim/sulfametoxazol) durante seis meses. Los autores encontraron que el número promedio de ITU fue de 0,36 en las pacientes vacunadas y de 1,6 en las tratadas con antibiótico $(p<0,0001)$. La reducción se observó también 9 y 15 meses después $(\mathrm{p}<0,0001)$. El número de pacientes que no volvió a tener una ITU fue de 101, 90 y 55 a los 3, 9 y 15 meses frente a 9, 4 y 0 , respectivamente $(\mathrm{p}<0,0001)$.

Más adelante, Lorenzo-Gómez et al. ${ }^{15}$ compararon retrospectivamente la eficacia de la vacuna MV
140-Uromune durante tres meses en 360 mujeres frente a otras 339 que tomaron la profilaxis antibiótica (40 $\mathrm{mg} / 200 \mathrm{mg}$ al día de trimetoprim/sulfametoxazol o 100 mg al día de nitrofurantoína) y al evaluar el intervalo de tiempo de aparición de una nueva ITU desde la intervención profiláctica encontraron que el $100 \%$ de las pacientes tratadas con antibiótico tuvieron una ITU, siendo 19 días el tiempo libre de infección (5-300), mientras que solo el 9,7 \% de las mujeres vacunadas presentaron esta condición ( $\mathrm{p}<0,0001)$.

Un tratamiento alternativo o concomitante al uso de la vacuna y/o la profilaxis antibiótica podría encontrarse en el fruto y las hojas del arándono rojo (Vaccinium macrocarpon); sin embargo, actualmente no existe suficiente evidencia que avale su uso en la prevención de la ITU recurrente. La evidencia que sustenta su empleo ha sido evaluada en una revisión sistemática ${ }^{16} \mathrm{y}$ en un ensayo clínico ${ }^{17}$ con faltas en sus diseños, pues ninguno de estos estudios alcanzó significación estadística en sus resultados. Por su parte, Beerepoot et al. ${ }^{18}$ analizaron 221 mujeres premenopáusicas con ITU recurrente, a quienes de manera aleatoria se les suministró una profilaxis antibiótica con 480 $\mathrm{mg}$ al día de trimetoprim/sulfametoxazol o $500 \mathrm{mg}$ cada 12 horas de arándano rojo durante 12 meses, y observaron una superioridad del antibiótico frente al arándano, con una incidencia de ITU del $71,1 \%$ y el $78,2 \%$, respectivamente. Dados estos resultados, hasta que no se disponga de más estudios prospectivos controlados con suficiente cantidad de ingrediente activo, medido de forma estandarizada, los productos de arándano rojo no pueden ser recomendados para la prevención de la ITU.

El presente trabajo es el primer estudio que evalúa la respuesta de la vacunación sublingual bacteriana en pacientes con ITU recurrente y ERC. Se trata de un estudio prospectivo en condiciones reales de práctica clínica que incluye pacientes con y sin ERC, y cuya frecuencia de ITU disminuye de manera significativa tras la administración de la vacuna durante tres meses. Este estudio realiza un seguimiento muy estrecho de los pacientes y un control exhaustivo de la existencia de episodios de ITU y su resolución. Cabe destacar que más de una cuarta parte de los participantes $(26,9 \%)$ no volvió a tener una infección de este tipo en los seis meses siguientes y casi la mitad $(46,1 \%)$

82 Vacunación sublingual con bacterias inactivadas en pacientes nefrológicos

con infección urinaria recurrente: experiencia en un centro de España

e2500-5006 Revista Colombiana de Nefrología 
tuvo menos episodios, lo cual repercute notablemente en la calidad de vida de los pacientes y en los costes asociados a las consultas médicas, la realización de urocultivos y la prescripción de antibioterapia. Llama la atención la mayor respuesta a la vacunación en pacientes con mejor función renal $(92,2 \%$ en sujetos sin ERC o con estadios I-II frente al $50 \%$ en pacientes con estadios IV-V, $\mathrm{p}=0,026$ ), lo que concuerda con la respuesta a otras vacunas, como la de la hepatitis B.

Sin embargo, este estudio tiene algunas limitaciones, como por ejemplo que carece de una muestra amplia de pacientes, es unicéntrico y no compara la eficacia de la vacunación con la profilaxis antibiótica, sino con la ausencia de una intervención. Por tanto, se precisa de estudios controlados que incluyan un mayor número de pacientes renales para corroborar los resultados aquí obtenidos. Dado que la vacunación puede realizarse hasta durante dos años, falta por determinar la respuesta de la ITU recurrente a la vacunación bacteriana a largo plazo y cuando se administra durante seis meses, así como la respuesta a un ciclo de vacunación y una dosis de recuerdo anual.

\section{Conclusión}

La vacunación sublingual bacteriana en pacientes nefrológicos con ITU recurrente es eficaz, especial- mente en los que presentan mejor función renal, pues el número medio de episodios de ITU se redujo significativamente después de la vacunación (3,62 vs. 1,69; $\mathrm{p}=0,001)$ : 19 pacientes tuvieron menos episodios, 5 pacientes siguieron igual y solo 2 sufrieron un mayor número de infecciones.

\section{Conflicto de intereses}

Ninguno declarado por los autores.

\section{Financiación}

Ninguna declarada por los autores.

\section{Agradecimientos}

Ninguno declarado por los autores.

\section{Contribución de los autores}

Por favor mencionar la contribución de cada uno de los autores en el manuscrito. 


\section{Referencias}

1. Vaqué J, Rodrigo JA. Prevalencia de las infecciones en los hospitales españoles. Estudio EPINE. Resultados de los estudios de 2004, 2005, 2006 y 2007 y evolución 1990-2007: 18 años. Resultados generales. Medicina Preventiva. 2008;14(1):1-75.

2. XAu H, Gasparini A, Ishigami J, Mzayen K, Su G, Barany P, et al. eGFR and the Risk of Community-Acquired Infections. Clin J Am Soc Nephrol. 2017. https://dx.doi.org/10.2215/CJN.00250117.

3. Sarnak MJ, Jaber BL. Mortality caused by sepsis in patients with end- stage renal disease compared with the general population. Kidney Int. 2000;58(4):1758-64. https://dx.doi.org/10.1111/j.1523-1755.2000.00337.x.

4. Naqvi SB, Collins AJ. Infectious complications in chronic kidney disease. Adv Chronic Kidney Dis. 2006;13(3):199-204. https://dx.doi.org/10.1053/j.ackd.2006.04.004.

5. Dalrymple LS, Katz R, Kestenbaum B, de Boer IH, Fried L, Sarnak MJ, et al. The risk of infection-related hospitalization with decreased kidney function. Am J Kidney Dis. 2012;59(3):356-63. https://dx.doi.org/10.1053/j.ajkd.2011.07.012.

6. James MT, Quan H, Tonelli M, Manns BJ, Faris P, Laupland KB, et al. CKD and risk of hospitalization and death with pneumonia. Am J Kidney Dis. 2009;54(1):24-32. https://dx.doi.org/10.1053/j.ajkd.2009.04.005.

7. Mc Donald HI, Thomas SL, Nitsch D. Chronic kidney disease as a risk factor for acute community-acquired infections in high-income countries: a systematic review. BMJ Open. 2014;4(4):e0044100. http://dx.doi.org/10.1136/bmjopen-2013-004100.

8. Cheikh-Hassan HI, Tang M, Djurdjev O, Langsford D, Sood MM, Levin A. Infection in advanced chronic kidney disease leads to increased risk of cardiovascular events, end-stage kidney disease and mortality. Kidney Int. 2016;90(4):897-904.

https://dx.doi.org/10.1016/j.kint.2016.07.013.

9. García Agudo R, Panizo N, Proy-Vega B, García-Martos P, Fernández-Rodríguez A. Infección del tracto urinario en la enfermedad renal crónica. Rev. Colomb. Nefrol. 2020;7(1):70-83. https://doi.org/10.22265/acnef.7.1.264.

10. Benito-Villalvilla C, Cirauqui C, Díez-Rivero CM, Casanovas M, Subiza JL, Palomares O. MV140, a sublingual polyvalent bacterial preparation to treat recurrent urinary tract infections, licenses human dendritic cells for generating Th1, Th17, and IL-10 responses via Syk and MyD88. Mucosal Immunol. 2016;10(4):924-935. https://dx.doi.org/10.1038/mi.2016.112.

11. Bauer HW, Rahlfs VW, Lauener PA, Blessmann GS. Prevention of recurrent urinary tract infections with immuno-active. E. coli fractions: a meta-analysis of five placebo-controlled double-blind studies. Int J Antimicrob Agents. 2002;19(6):451-6. https://dx.doi.org/10.1016/s0924-8579(02)00106-1.

12. Bauer HW, Alloussi S, Egger G, Blümlein HM, Cozma G, Schulman CC. A long-term, multicenter, double-blind study of an Escherichia coli extract (OM-89) in female patients with recurrent urinary tract infections. Eur Urol. 2005;47(4):542-8. https://dx.doi.org/10.1016/j.eururo.2004.12.009.

13. Naber KG, Cho YH, Matsumoto T, Schaeffer AJ. Immunoactive prophylaxis of recurrent urinary tract infections: a meta-analysis. Int J Antimicrob Agents 2009;33(2):111-9. https://dx.doi.org/10.1016/j.ijantimicag.2008.08.011.

14. Lorenzo-Gómez MF, Padilla-Fernández B, García-Criado FJ, Mirón-Canelo JA, Gil-Vicente A, Nieto-Huertos A, et al. Evaluation of a therapeutic vaccine for the prevention of recurrent urinary tract infections versusprophylactic treatment with antibiotics. Int Urogynecol J. 2013;24(1):127-34. https://dx.doi.org/10.1007/s00192-012-1853-5.

15. Lorenzo-Gómez MF, Padilla-Fernández B, García-Cenador MB, Virseda- Rodríguez AJ, Martín-García I, Sánchez-Escudero A, et al. Comparison of sublingual therapeutic vaccine with antibiotics for the prophylaxis of recurrent uninary tract infections. Front Cell Infect Microbiol. 2015;5:50. https://dx.doi.org/10.3389/fcimb.2015.00050.

16. Jepson RG, Williams G, Craig JC. Cranberries for preventing urinary tract infections. Cochrane Database Syst Rev. 2012;10(10):CD001321. https://dx.doi.org/10.1002/14651858.CD001321.pub5.

17. Stapleton AE, Dziura J, Hooton TM, Cox MB, Yarova-Yarovaya Y, Chen S, et al. Recurrent urinary tract infection and urinary Escherichia coli in women ingesting cranberry juice daily: a randomized controlled trial. Mayo Clinic Proc. 2012;87(2):143-50. https://dx.doi.org/10.1016/j.mayocp.2011.10.006.

18. Beerepoot MA, ter Riet G, Nys S, van der Wal WM, de Borgie CA, de Reijke TM, et al. Cranberries vs antibiotics to prevent urinary tract infections: a randomized double-blind noninferiority trial in premenopausal women. Arch Intern Med. 2011;171(14):1270-8. https://dx.doi.org/10.1001/archinternmed.2011.306.

84 Vacunación sublingual con bacterias inactivadas en pacientes nefrológicos con infección urinaria recurrente: experiencia en un centro de España 